\title{
Longitudinal Coherence Preservation and Chirp Evolution in a High Gain Laser Seeded Free Electron Laser Amplifier*
}

\author{
J.B. Murphy, ${ }^{1}$ Juhao $\mathrm{Wu}^{2, \dagger}$ X.J. Wang, ${ }^{1}$ and T. Watanabe ${ }^{1}$ \\ ${ }^{1}$ National Synchrotron Light Source, Brookhaven National Laboratory, Upton, NY 11973-5000 \\ 2 Stanford Linear Accelerator Center, Menlo Park, CA 94025
}

(Dated: May 05, 2006)

\begin{abstract}
In this letter we examine the start-up of a high gain free electron laser in which a frequencychirped coherent seed laser pulse interacts with a relativistic electron beam. A Green function formalism is used to evaluate the initial value problem. We have fully characterized the startup and evolution through the exponential growth regime. We obtain explicit expressions for the pulse duration, bandwidth and chirp of the amplified light and show that the FEL light remains fully longitudinally coherent.
\end{abstract}

\section{Submitted to Physical Review Letters}

PACS numbers: 41.60.Cr, 42.25.Kb

The free electron laser (FEL) makes use of a periodic magnetic field (undulator) to transform the kinetic energy of a relativistic electron beam into electromagnetic radiation. The FEL has the potential to provide tunable "laser-like" photon beams in short wavelength (xray) regimes that are inaccessible by quantum lasers[1]. It is imperative to understand how well an FEL can reproduce some of the key laser-like properties such as longitudinal coherence, chirped pulses and bandwidth control. We will examine these properties for a laser seeded free electron laser.

Several authors have examined the initial value problem using a Green function formalism for the start-up of a high gain free electron laser from shot noise, the so-called Self Amplified Spontaneous Emission (SASE) solution [2-7]. In this letter we examine the start-up of a high gain free electron laser in which a frequency-chirped coherent seed laser pulse interacts with a relativistic electron beam. A Green function formalism is used to evaluate the initial value problem. We examine the evolution of the bandwidth, pulse duration, chirp and longitudinal coherence of a laser seeded FEL.

We begin with a discussion of the frequency-chirped Gaussian seed laser pulse. The electric field of the seed laser is assumed to be,

$$
E_{s}(t, z)=E_{0} e^{i\left(k_{0} z-\omega_{0} t\right)} e^{-(\alpha+i \beta)(t-z / c)^{2}},
$$

where $\omega_{0}, k_{0}, \alpha$ and $\beta$ characterize the pulse frequency, wavenumber, duration, and chirp, respectively. To fully characterize the longitudinal properties of the seed pulse jointly in both the time and frequency domains requires the introduction of the Wigner distribution function

\footnotetext{
*Work supported by the U.S. Department of Energy under contract number DE-AC02-98CH10886 and the Office of Naval Research (JBM, XJW, TW) and by the U.S. Department of Energy under contract number DE-AC02-76SF00515 (JW)

${ }^{\dagger}$ Electronic address: jhwu@SLAC.Stanford.EDU
}

which is defined as follows [8, 9],

$$
W(t, \omega, z)=\int E(t-\tau / 2, z) E^{*}(t+\tau / 2, z) e^{-i \omega \tau} d \tau
$$

For the chirped Gaussian seed pulse of Eq. (1) the Wigner distribution function is given by,

$$
\begin{aligned}
W_{s}(t, \omega, z) & =\left|E_{0}\right|^{2} \sqrt{\frac{2 \pi}{\alpha}} \exp \left\{-\left[4\left(t-\frac{z}{c}\right)^{2}\left(\alpha^{2}+\beta^{2}\right)(3)\right.\right. \\
& \left.\left.-4 \beta\left(t-\frac{z}{c}\right)\left(\omega-\omega_{0}\right)+\left(\omega-\omega_{0}\right)^{2}\right] /(2 \alpha)\right\} .
\end{aligned}
$$

The average values of $t, \omega$, the rms pulse duration, the rms bandwidth and the cross moment of the seed laser can be determined as moments of the Wigner function,

$$
\left\{\begin{array}{l}
\langle t\rangle=\frac{z}{c}=\frac{z}{v_{g}} \\
\langle\omega\rangle=\omega_{0} \\
\sqrt{\left\langle(t-\langle t\rangle)^{2}\right\rangle}=\sigma_{t, \text { seed }}=\frac{1}{2 \sqrt{\alpha}} \\
\sqrt{\left\langle(\omega-\langle\omega\rangle)^{2}\right\rangle}=\sigma_{\omega, \text { seed }}=\sqrt{\frac{\alpha^{2}+\beta^{2}}{\alpha}} \\
\langle(t-\langle t\rangle)(\omega-\langle\omega\rangle)\rangle=\frac{\beta}{2 \alpha}
\end{array} .\right.
$$

In the above we have introduced the group velocity of the pulse which in a vacuum is simply $v_{g}=c$. It will be seen later that the FEL interaction reduces the group velocity of the amplified seed laser pulse.

The longitudinal emittance of a light beam is defined as,

$$
\varepsilon_{\text {Light }} \equiv \sqrt{\left\langle(t-\langle t\rangle)^{2}\right\rangle\left\langle(\omega-\langle\omega\rangle)^{2}\right\rangle-\langle(t-\langle t\rangle)(\omega-\langle\omega\rangle)\rangle^{2}} .
$$

Substituting for the moments from Eq. (4) it can be shown that $\varepsilon_{\text {Light }}=1 / 2$, the emittance of the seed light beam is a constant and equal to the minimum uncertainty value as determined by the Heisenberg uncertainty relation, $\Delta t \Delta \omega \geq 1 / 2$, where the rms values are used for $\Delta t \equiv \sigma_{t}$ and $\Delta \omega \equiv \sigma_{\omega}$.

This means that the light beam is fully longitudinally coherent, consisting of a single longitudinal mode, for all 
values of the chirp parameter. In this letter, we will show that when the seed laser is amplified in the FEL it remains fully longitudinally coherent throughout the exponential gain regime. We will also consider the evolution of the chirp and bandwidth of the amplified pulse.

To analyze the start-up of a seeded FEL amplifier we must use the coupled set of Vlasov and Maxwell equations which describe the evolution of the particles and the radiation fields. Since we are only interested in the longitudinal properties of the radiation field we will ignore diffraction effects inherent in a three dimensional description and limit ourselves to a one dimensional model. We follow the analysis and notation of Ref. [6]. Even though, we study a seeded FEL in this letter, part of the derivation is the same as for the SASE case. We start from the point where the derivation for the seeded FEL is different from those for SASE FEL.

For the seeded FEL, we start with Eq. (A9) of Ref. [6], but set $\mu=0$ as our electron beam is not chirped, and keep only the $A\left(\theta^{\prime}, 0\right)$ term to get,

$$
f(\theta, s)=\int_{-\infty}^{\theta} d \theta^{\prime} e^{-s\left(\theta-\theta^{\prime}\right)+\frac{i(2 \rho)^{3}\left(\theta-\theta^{\prime}\right)}{s^{2}}} A\left(\theta^{\prime}, 0\right),
$$

where $A(\theta, Z)$ is the slow varying envelope function, i.e., the light field is $E(t, z)=A(\theta, Z) e^{i(\theta-Z)}$ with dimensionless variables $Z=k_{w} z, \theta=\left(k_{0}+k_{w}\right) z-\omega_{0} t$, and $k_{w}=2 \pi / \lambda_{w}$ with $\lambda_{w}$ being the undulator period. The Laplace transform pair, $f(\theta, s)$ and $A(\theta, Z)$, is defined by

$$
\left\{\begin{array}{l}
f(\theta, s)=\int d Z e^{-s Z} A(\theta, Z) \\
A(\theta, Z)=\int_{c} \frac{d s}{2 \pi i} e^{s Z} f(\theta, s)
\end{array} .\right.
$$

Hence,

$$
\begin{array}{r}
A(\theta, Z)=\int_{c^{2}} \frac{d s}{2 \pi i} e^{s Z} \int_{-\infty}^{\theta} d \theta^{\prime} e^{-s\left(\theta-\theta^{\prime}\right)+\frac{i(2 \rho)^{3}\left(\theta-\theta^{\prime}\right)}{s^{2}}} A\left(\theta^{\prime}, 0\right) \\
\cong e^{\rho(\sqrt{3}+i) Z} \int_{0}^{\infty} d \xi e^{-\rho(\sqrt{3}+i)\left[9(\xi-Z / 3)^{2} /(4 Z)\right]} A(\theta-\xi, 0) .(8)
\end{array}
$$

Once the seed input, $A(\theta, 0)$, is specified, we can obtain the seeded FEL $A(\theta, Z)$, along the undulator.

Let us now work with a Gaussian seed as in Eq. (1),

$$
E(z=0, t)=e^{-i \omega_{0} t-(\alpha+i \beta) t^{2}}=e^{i \theta-\frac{\theta^{2}}{\omega_{0}^{2}}(\alpha+i \beta)},
$$

which gives the slow varying envelope function

$$
A(\theta, 0)=e^{-\frac{\theta^{2}}{\omega_{0}^{2}}(\alpha+i \beta)} .
$$

Therefore,

$$
\begin{aligned}
A(\theta, Z) & \cong e^{\rho(\sqrt{3}+i) Z} \int_{0}^{\infty} d \xi e^{-\frac{(\theta-\xi)^{2}}{\omega_{0}^{2}}(\alpha+i \beta)-\rho(\sqrt{3}+i)\left[9(\xi-Z / 3)^{2} /(4 Z)\right]} \\
& \cong e^{\frac{3}{4} \rho(\sqrt{3}+i) Z-\frac{\theta^{2}}{\omega_{0}^{2}}(\alpha+i \beta)-\frac{\left\{\frac{4 i \theta}{\omega_{0}^{2}}(\alpha+i \beta)+3 i \rho(\sqrt{3}+i)\right\}^{2}}{\frac{16 Z}{\omega_{0}^{2}}(\alpha+i \beta)+36 \rho(\sqrt{3}+i)}} Z \text {. (11) }
\end{aligned}
$$

Rewriting the electric field in the $(t, z)$ coordinates gives,

$$
\begin{aligned}
E_{\mathrm{FEL}}(t, z) & =E_{0, \mathrm{FEL}} e^{\rho(\sqrt{3}+i) k_{w} z} \\
& \times e^{i\left(k_{0} z-\omega_{0} t\right)} e^{-[\alpha(z)+i \beta(z)]\left(t-z / v_{g}\right)^{2}},
\end{aligned}
$$

where

$$
v_{g}=\frac{\omega_{0}}{k_{0}+\frac{2}{3} k_{w}}
$$

is the group velocity of the amplified FEL light[10, 11]. Introducing

$$
\sigma_{\omega, \mathrm{GF}}(z) \equiv \sqrt{\frac{3 \sqrt{3} \rho \omega_{0}^{2}}{k_{w} z}}
$$

which is the rms bandwidth of the FEL Green function for a coasting electron beam [2-4], and computing moments of the Wigner function for the field in Eq. (12), yields $\alpha(z)=1 /\left[4 \sigma_{t}(z)^{2}\right]$ and $\beta(z)^{2}=\sigma_{\omega}(z)^{2} \alpha(z)-\alpha(z)^{2}$ with

$$
\left\{\begin{array}{l}
\sigma_{t}(z)=\sqrt{\frac{4 \sigma_{\omega, \mathrm{seed}}^{2}+\left(6+2 \sqrt{3} \frac{\beta}{\alpha}\right) \sigma_{\omega, \mathrm{GF}}^{2}(z)+\frac{3}{\alpha} \sigma_{\omega, \mathrm{GF}}^{4}(z)}{12 \sigma_{\omega, \mathrm{GF}}^{2}(z)\left[\sigma_{\omega, \text { seed }}^{2}+\sigma_{\omega, \mathrm{GF}}^{2}(z)\right]}} \\
\sigma_{\omega}(z)=\sqrt{\frac{1}{\frac{1}{\sigma_{\omega, \text { seed }}^{2}+\frac{1}{\sigma_{\omega, \mathrm{GF}}^{2}(z)}}}}
\end{array}\right.
$$

and

$$
\begin{aligned}
\langle(t-\langle t\rangle)(\omega-\langle\omega\rangle)\rangle & \equiv \frac{\beta(z)}{2 \alpha(z)} \\
& =\frac{1}{2 \sqrt{3}} \frac{\sigma_{\omega, \text { seed }}^{2}+\sqrt{3} \frac{\beta}{\alpha} \sigma_{\omega, \mathrm{GF}}^{2}(z)}{\sigma_{\omega, \text { seed }}^{2}+\sigma_{\omega, \mathrm{GF}}^{2}(z)}
\end{aligned}
$$

It is important to note that in the limit $z \rightarrow 0$, the above expressions for the moments reduce to the expressions for the seed laser alone as given in Eq. (4). Since we are only interested in the exponential growth regime, we will not specify the initial value $E_{0, \mathrm{FEL}}$.

The emittance of the FEL light at any position, $z$, along the undulator is defined in the same fashion as in Eq. (5) but now the $z$-dependent moments derived above must be used to obtain, $\varepsilon_{\text {Light }}(z)=1 / 2$. The constancy of the emittance means that the amplified seed laser or FEL light remains fully longitudinally coherent throughout the exponential gain regime.

It can be seen from the formulas above or Figs. 1 and 2 that the phase space begins with the characteristics of the seed laser and evolves as the FEL interaction reduces the bandwidth monotonically, modifies the pulse duration and imposes its own chirp on the amplified light. This evolution occurs in a fashion that preserves the area of the phase space ellipse and hence preserves the longitudinal coherence of the amplified seed light. In Figs. 1 and 2, the Wigner function has the same formal expression as in Eq. (3), with $\alpha \rightarrow \alpha(z), \beta \rightarrow \beta(z)$, and $c \rightarrow v_{g}$ given in Eq. (13)-(16).

The complex evolution of the phase space ellipse gives rise to the interesting possibility that even though the 

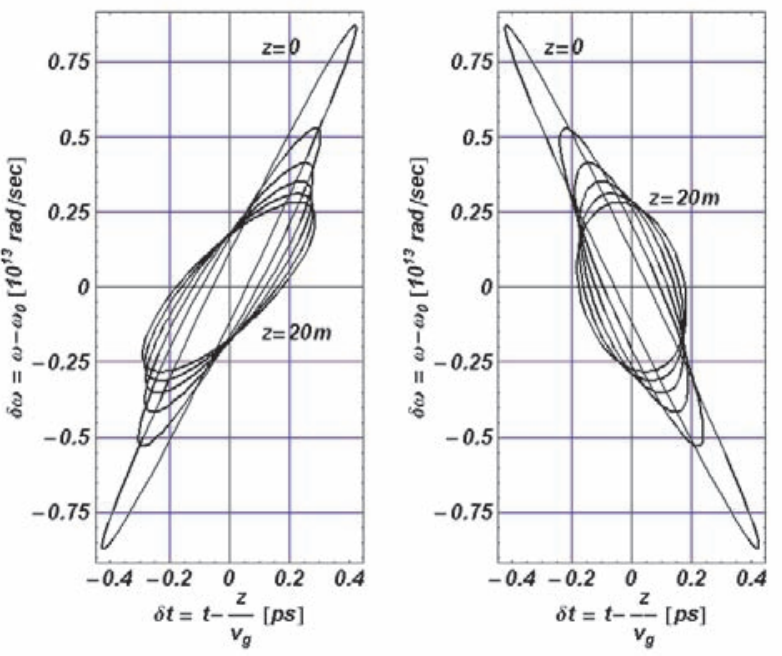

FIG. 1: Contour plot of the Wigner function $W\left(t-z / v_{g}, \omega-\right.$ $\left.\omega_{0}\right)$ of the FEL light for $z \in(0,20) \mathrm{m}$ and with $\rho=10^{-3}$, $\lambda_{0}=0.8 \mu \mathrm{m}$ and $\lambda_{w}=0.039 \mathrm{~m}$. The group velocity $v_{g}$ is given in Eq. (13). The left/right subplot is for an initial positive/negative seed laser chirp of equal magnitude.

bandwidth decreases monotonically, the pulse duration can also decrease initially before it eventually grows. This can be seen in Fig. 2 which displays the pulse duration, bandwidth, chirp, and longitudinal emittance versus the distance along the undulator for a realistic set of FEL parameters $\left(\rho=10^{-3}, \lambda_{0}=0.8 \mu \mathrm{m}\right.$ and $\lambda_{w}=0.039$ $\mathrm{m})$. Note that even in the absence of an initial chirp of the seed laser the FEL will impose a chirp on the amplified light. Note that the FEL bandwidth in Eq. (15) is independent of the sign of the chirp in the seed laser so the two curves for $\sigma_{\omega}(z)$ in Fig. 2 lie on top of each other since the initial chirp is of opposite sign but of the same magnitude. It should be noted that, in the initial stage, even though both the pulse duration and the bandwidth are reducing, the chirp is reducing accordingly to preserve the longitudinal emittance. The phase space evolution must be examined in detail for the particular input conditions for each FEL.

The analysis in this manuscript is limited to the exponential gain regime before saturation. In general, for an FEL amplifier the output pulse duration, bandwidth and chirp will depend not only on the seed pulse duration, bandwidth and chirp but also on the seed amplitude as the amount of evolution of the phase space depends on how close to saturation the seed laser pulse is when it is injected into the FEL.

A gauge of the limitations can be obtained by considering the well known SASE results which defines a cooperation or coherence length as [2],

$$
L_{c, \mathrm{SASE}}(z)=\frac{c}{\sigma_{\omega, \mathrm{GF}}(z)} .
$$

The SASE cooperation length grows from zero to a maximum value determined by the undulator length it takes
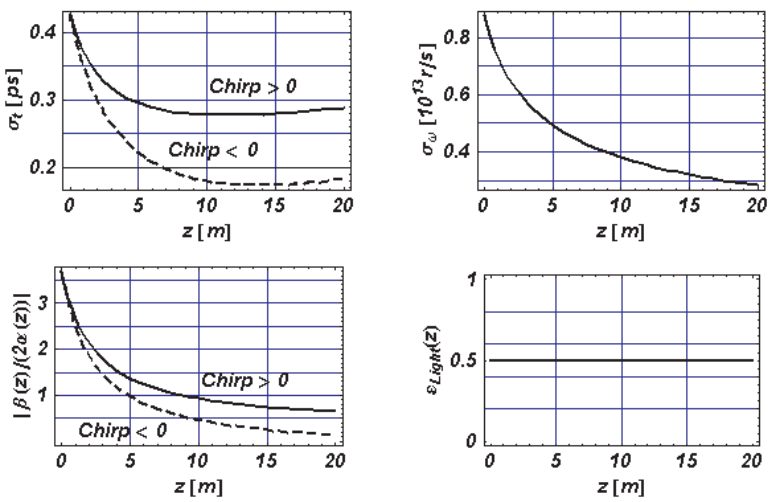

FIG. 2: Plot of the FEL pulse duration, bandwidth, chirp, and longitudinal emittance versus $z$ for the same conditions as in Fig. 1. Chirp $>0$ corresponds to the left side figure in Fig. 1.

for the SASE process to saturate, which is approximately 20 power gain lengths, $z_{\text {sat }} \simeq \lambda_{w} / \rho$. At saturation the SASE cooperation length and bandwidth are given by,

$$
\left\{\begin{array}{l}
L_{c, \operatorname{SASE}}\left(z_{\mathrm{sat}}\right) \approx \frac{\lambda}{2 \pi \rho} \\
\frac{\sigma_{\omega, \operatorname{SASE}}\left(z_{\mathrm{sat}}\right)}{\omega_{0}} \approx \rho
\end{array} .\right.
$$

In order to justify ignoring SASE as we have done, the seeded FEL must saturate well before SASE so that the minimum bandwidth for the seeded FEL must satisfy the following,

$$
\frac{\sigma_{\omega}\left(z_{\mathrm{sat}}\right)}{\omega_{0}} \geq \frac{\rho}{\sqrt{\frac{\rho^{2}}{\frac{\sigma_{\omega, \text { seed }}^{2}}{\omega_{0}^{2}}}+1}} .
$$

The cooperation or coherence length for the seeded FEL is defined in a similar fashion to that for SASE as the inverse of the bandwidth,

$$
L_{c}(z)=\frac{c}{\sigma_{\omega}(z)}=c \sqrt{\frac{1}{\sigma_{\omega, \text { seed }}^{2}}+\frac{1}{\sigma_{\omega, \mathrm{GF}}^{2}(z)}} .
$$

In contrast to SASE, in this case the cooperation length begins with the value determined from the seed laser which has a coherent phase and increases monotonically due to the slippage of the radiation pulse relative to the electron beam.

There are several velocities of interest in an FEL, which are related to either the radiation field or the electron beam. In free space (vacuum) we have the phase and group velocities of the radiation pulse (wavepacket):

$$
v_{\phi, \mathrm{vac}}=v_{g, \mathrm{vac}}=\frac{\omega_{0}}{k_{0}}=c .
$$

The resonant FEL interaction is defined as synchronism between the electron's axial velocity and the phase velocity of the pondermotive wave:

$$
v_{z}=\frac{\omega_{0}}{k_{0}+k_{w}} .
$$


Since the free space radiation travels faster than the electron, in the time it takes the electron to traverse the full undulator the electron slips behind the free space radiation as follows:

$$
S_{\mathrm{vac}}=\left(v_{g, \mathrm{vac}}-v_{z}\right) \frac{N_{w} \lambda_{w}}{v_{z}}=N_{w} \lambda_{0} .
$$

The FEL interaction modifies the phase and group of the radiation wavepacket as follows:

$$
\left\{\begin{array}{l}
v_{\phi, \mathrm{FEL}}=\frac{\omega_{0}}{k_{0}+\rho k_{w}} \\
v_{g, \mathrm{FEL}}=\frac{\omega_{0}}{k_{0}+\frac{2}{3} k_{w}}
\end{array}\right.
$$

The relative ordering of all these velocities is:

$$
v_{z}<v_{g, \mathrm{FEL}}<v_{\phi, \mathrm{FEL}}<v_{g, \mathrm{vac}}=v_{\phi, \mathrm{vac}}=c .
$$

Since the FEL interaction reduces the group velocity of the radiation wavepacket, the slippage of the electron relative to the radiation wavepacket is reduced:

$$
S_{\mathrm{FEL}} \approx\left(v_{g, \mathrm{FEL}}-v_{z}\right) \frac{N_{w} \lambda_{w}}{v_{z}} \approx \frac{N_{w} \lambda_{0}}{3}
$$

To obtain an understanding of why the input pulse maintains its longitudinal coherence during the exponential growth regime one can examine the form of the integral equation in Eq. (8) which propagates an input pulse through the FEL via the Green function. The convolution integral is of the general form of an integral representation of an $A B C D$ canonical transformation, as such the phase space area and longitudinal coherence is preserved $[12,13]$.

Associated with the canonical transformation is a symplectic $A B C D$ matrix [12-14],

$$
M_{A B C D}=\left(\begin{array}{cc}
A & B \\
C & D
\end{array}\right)=\left(\begin{array}{cc}
1 & -\frac{2 i k_{w} z}{9(i+\sqrt{3}) \rho \omega_{0}} \\
0 & 1
\end{array}\right)
$$

The form of the $A B C D$ matrix $(A=D=1, C=0)$ and $B$ complex is characteristic of a system with group velocity dispersion $(\operatorname{Re} B)$ and gain $(\operatorname{Im} B)$. Note that the $A B C D$ matrix can be decomposed into a product of a pair of commuting matrices for the group velocity dispersion and the gain. The group velocity dispersion is responsible for the "horizontal shearing" of the phase space ellipse in Fig. 1, this together with the bandwidth reduction results in the initial compression of the chirped seed pulse. Since $C=0$ there is no inherent time lensing or pure chirping effect ("vertical shearing") in the FEL [15], an input pulse changes its overall chirp due to the group velocity dispersion and bandwidth reduction. It's possible to start with a negatively chirped seed and exit the FEL with no chirp.

The $A B C D$ matrix can be used to transform the complex Gaussian pulse parameter, $p(z)$ [14],

as follows

$$
\frac{1}{p(z)} \equiv-\frac{2 \beta(z)}{\omega_{0}}+i \frac{2 \alpha(z)}{\omega_{0}},
$$

$$
p(z)=\frac{A p(0)+B}{C p(0)+D},
$$

to obtain the new chirp and pulse duration after the FEL interaction as given in Eqs. (15) and (16) confirming the results computed as moments of the Wigner function.

The $A B C D$ analysis is not limited to a simple chirped Gaussian seed pulse as given in Eq. (1). Arbitrary seed pulses can be constructed from a complete set of chirped Hermite-Gaussian functions characterized by a single $p$-parameter and propagated through the system with the $A B C D$ matrix formalism [14].

In this letter, we have fully characterized the startup and evolution through the exponential growth regime of the field of an FEL amplifier in which a coherent frequency-chirped seed pulse interacts with a relativistic electron beam. We obtained expressions for the pulse duration, bandwidth and chirp of the amplified light and we have shown that the light remains fully longitudinally coherent.

The work of JBM, XJW, and TW was supported by the US Department of Energy under contract DE-AC0298CH10886 and the Office of Naval Research. The work of JW was supported by the US Department of Energy under contract DE-AC02-76SF00515.
[1] J. Arthur et al., Linac Coherent Light Source Design Study Report, SLAC Report No. SLAC-R-521 (1998); R. Brinkman et al., TESLA XFEL: First Stage of the XRay Laser Laboratory, TESLA Report No. TESLA FEL 2002-09 (2002).

[2] J.-M. Wang and L.-H. Yu, Nuc. Instrum. and Methods in Physics Research A 250, 484 (1986).

[3] K.J. Kim, Nuc. Instrum. and Methods in Physics Research A 250, 396 (1986).

[4] K.J. Kim, Phys. Rev. Lett. 57, 1871 (1986).

[5] K.J. Kim, LBNL Report No. 40672 (1997).

[6] S. Krinsky and Z. Huang, Phys. Rev. ST Accel. Beams
6, 050702 (2003).

[7] E.L. Saldin, E.A. Schneidmiller, and M.V. Yurkov, Proceedings of the 27th International Free Electron Laser Conference, 258 (2005).

[8] E. Wigner, Phys. Rev. 40, 749 (1932).

[9] M.J. Bastiaans, Optik 82, 173 (1989).

[10] R. Bonifacio, L. DeSalvo Souza, P. Pierini, and N. Piovella, Nuc. Instrum. and Methods in Physics Research A 296, 358 (1990).

[11] G.T. Moore and N. Piovella, IEEE Jour. Quantum Elec. 27, 2522 (1991).

[12] K.B. Wolf, Integral Transforms in Science and Engineer- 
ing, Plenum (1979).

[13] R. Ortega-Martinez, C.J. Roman-Moreno, and A.L. Rivera, Rev. Mex. de Fisica 48, 565 (2002).

[14] S.P. Dijaili, A. Dienes, and J.S. Smith, IEEE Jour. Quan- tum Elec. 26, 1158 (1990).

[15] B.H. Kolner, IEEE Jour. Quantum Elec. 30, 1951 (1994). 